\title{
Banco de semillas del suelo en un pastizal de una reserva natural del Sistema de Tandilia (Buenos Aires, Argentina) invadido por Racosperma melanoxylon
}

\author{
Osvaldo R. Vignolio ${ }^{1, \bigotimes}$; María E. Garavano ${ }^{1}$; Patricia Diez de Ulzurrun ${ }^{1}$; \\ Hernán P. Angelini ${ }^{2}$ \& Verónica N. Ispizúa ${ }^{1}$
}

${ }^{1}$ Facultad de Ciencias Agrarias, Universidad Nacional de Mar del Plata. Balcarce, Argentina. ${ }^{2}$ INTA, Centro Regional Buenos

\author{
Aires Sur, EEA Balcarce. Balcarce, Argentina.
}

\begin{abstract}
Resumen. La restauración de los pastizales naturales invadidos por especies vegetales exóticas depende de la composición del banco de semillas del suelo. Se analizó la composición del banco de semillas germinable del suelo en dos ambientes de la Reserva Natural Paititi (Buenos Aires, Argentina): pastizales de Paspalum quadrifarium Lam. (pastizal natural) y pastizales invadidos por árboles exóticos que no superaban los cinco años de edad, Racosperma melanoxylon (R. Br.) Pedley, sinónimo de Acacia melanoxylon (pastizal invadido). Se tomaron muestras de suelo de cada ambiente en tres sitios, y mediante el registro de la emergencia de plántulas bajo condiciones controladas se determinó: 1) la densidad del banco de semillas total y de los grupos funcionales (dicotiledóneas, gramíneas y graminoides), la riqueza, la equitatividad, la diversidad y la abundancia específica, 2) la disimilitud florística, y 3) el valor zootécnico. Ni el banco de semillas de las especies nativas ni el de las exóticas difirieron significativamente entre ambientes. La densidad promedio del banco de semillas y de la riqueza no fueron significativamente diferentes entre ambientes $\left(\sim 10000 \mathrm{semillas} / \mathrm{m}^{2}\right.$ y 30 especies, respectivamente). El número de semillas de los grupos funcionales y los índices de diversidad y de equitatividad tampoco fueron significativamente diferentes entre ambientes. Sin embargo, la disimilitud florística fue 0.36 y se explica por la ausencia de un mayor número de especies en el pastizal invadido, entre ellas, las buenas forrajeras. Esto determinó un valor zootécnico $26 \%$ menor en el pastizal invadido que en el natural. En el pastizal invadido, todas las plantas de $P$. quadrifarium estaban muertas y su banco de semillas germinable fue casi un tercio que en el pastizal natural. Estos resultados permiten considerar que si se eliminan los árboles de R. melanoxylon del pastizal recientemente invadido se podría conservar un banco de semillas comparable al del pastizal natural.
\end{abstract}

[Palabras clave: Paspalum quadrifarium, árboles invasores, valor zootécnico, Acacia melanoxylon]

\begin{abstract}
Aвstract. Soil seed bank study in a grassland of a natural reserve of the Tandilia System (Buenos Aires, Argentina) invaded by Racosperma melanoxylon. The restoration of natural grasslands invaded by exotic plant species depends on the composition of the soil seed bank. We analyzed the composition of germinable soil seed bank in two environments of the Paititi Natural Reserve (Buenos Aires, Argentina): Paspalum quadrifarium Lam. grasslands (natural grassland) and grasslands invaded by species of exotic trees that were no more than five years old, Racosperma melanoxylon (R. Br.) Pedley, synonymous Acacia melanoxylon (invaded grassland). Soil samples from each environment were taken at three sites, and by recording seedling emergence under controlled conditions we determined: 1) the total soil seed bank density of the functional groups (dicots, grasses and graminoids), richness, equitability and diversity indices, and specific abundance, 2) floristic dissimilarity, and 3) zootechnical value. The seed bank of native and exotic species was not significantly different between environments. Average seed bank density and species richness were not significantly different between environments ( 10000 seeds $/ \mathrm{m}^{2}$ and 30 species, respectively). Seed density of the functional groups and the diversity and equitability indices were not significantly different between environments. Still, floristic dissimilarity among environments was as low as 0.36 , explained by the absence of a greater number of species in the invaded grassland. The seed bank of invaded grasslands was poor in forage species, consistent with a zootechnical value $26 \%$ lower than in the natural grassland. In the invaded grassland all $P$. quadrifarium plants were dead and their germinable seed bank was a third than in the natural grassland. The results suggest that an early intervention removing $R$. melanoxylon trees in the grassland invaded may preserve a soil seed bank comparable to those uninvaded grassland.
\end{abstract}

[Keywords: Paspalum quadrifarium, invasive trees, zootechnical value, Acacia melanoxylon]

Editor asociado: Fernando Milesi ovignoli@mdp.edu.ar
Recibido: 23 de Septiembre de 2020

Aceptado: 20 de Abril de 2021 


\section{INTRODUCCIÓN}

Las especies invasoras pueden modificar la estructura, el funcionamiento y la capacidad de los ecosistemas de proveer servicios; es decir, los beneficios que brindan para el bienestar de la sociedad (Mack et al. 2000; Pimentel 2002; Le Maitre et al. 2011; Sala et al. 2017). Algunas de las especies del género Acacia son una amenaza para la biodiversidad de los ecosistemas que invaden porque establecen extensas formaciones monoespecíficas que excluyen a la vegetación nativa (Mack et al. 2000; Le Maitre et al. 2011). Algunos de los atributos del género Acacia que explican el éxito de su invasión son: a) liberación de sustancias alelopáticas que afectan de forma negativa el tamaño del banco de semillas y la germinación de las especies nativas, b) acumulación de biomasa en el suelo que actúa como una barrera física para el establecimiento de las plántulas, c) exclusión de las especies residentes por competencia aérea y subterránea, y d) las raíces de las plantas pueden tomar los nutrientes y el agua de zonas más profundas que las nativas (Pimentel 2002; Ferrari and Wall 2004; Hussain et al. 2011; Le Maitre et al. 2011; Renuka et al. 2012; Souza-Alonso et al. 2017).

En los ecosistemas serranos del Sistema de Tandilia (Buenos Aires, Argentina), una de las especies exóticas arbóreas que se ha propagado con éxito es la Acacia melanoxylon R. Br., actualmente clasificada como Racosperma melanoxylon (R. Br.) Pedley (Fabaceae, Mimosoideae) (a partir de aquí, R. melanoxylon o acacia). A esta especie se la considera naturalizada en la zona de las sierras de las ciudades de Balcarce y Azul (Buenos Aires, Argentina) desde aproximadamente 1947 (Carranza 2017). En la Región Pampeana (Buenos Aires), los pastizales naturales en buen estado de conservación están restringidos a los ecosistemas donde la frontera agropecuaria no ha podido avanzar, como los afloramientos rocosos de las sierras, los humedales, los suelos con drenaje pobre y las dunas de la franja costera (Zalba and Villamil 2002; Baldi et al. 2006). Sin embargo, la invasión de árboles exóticos es una amenaza para algunos de estos ecosistemas (Ghersa et al. 2002; Zalba and Villamil 2002). Este es el caso de la Reserva Natural Paititi (RNP), un ecosistema serrano que forma parte del Sistema de Tandilia, cuyos pastizales, dominados por Paspalum quadrifarium Lam. (una gramínea nativa $\mathrm{C}_{4}$ ), están invadidos por plantas de $R$. melanoxylon (Echeverría et al. 2017; Garavano
2018; Arcusa 2019). La RNP está ubicada en el partido de General Pueyrredón y es refugio de biodiversidad de la flora y la fauna nativa (Echeverría et al. 2017; Garavano 2018; Arcusa 2019). Los pastizales naturales de la RNP se usan como recurso forrajero para vacunos y equinos (Garavano 2018), quedando el área de pastoreo restringida a las zonas no invadidas. Los registros de la flora realizados por Garavano (2018) en los pastizales de la RNP nos permiten confirmar que la propagación de las plantas de R. melanoxylon coincidió con un incendio en el verano del año 2014. Al eliminar la vegetación herbácea del pastizal, este tipo de disturbio modifica la temperatura, la luz y la humedad del suelo, condiciones que explican la rápida invasión de $R$. melanoxylon (Le Maitre et al. 2011). Un caso comparable al de la RNP es que documentaron Cuevas y Zalba (2013) acerca de la invasión de una especie exótica, Pinus halepensis Mill., en el Parque Provincial Ernesto Tornquist (Sierra de la Ventana, Buenos Aires, Argentina) luego de un incendio en los pastizales naturales.

Una práctica para restablecer los pastizales naturales en reservas invadidas por árboles exóticos es el corte de las plantas, combinado - en algunos casos - con el control químico de los rebrotes (Le Maitre et al. 2011; Cuevas and Zalba 2013). Sin embargo, si se eliminan los árboles, la capacidad de la comunidad vegetal de retornar naturalmente a la estructura original dependerá del tiempo de invasión, del tamaño del banco de semillas y de los órganos de propagación vegetativa de las especies nativas (Le Maitre et al. 2011; Cuevas and Zalba 2013; Souza-Alonso et al. 2017). En otras palabras, un tiempo prolongado de invasión puede producir cambios irreversibles en la estructura y el funcionamiento de la comunidad vegetal y, en tal sentido, las prácticas tempranas de control aumentarán las probabilidades de restauración del pastizal natural (Amiotti et al. 2000; Zalba and Villamil 2002; Cuevas and Zalba 2010, 2013; Le Maitre et al. 2011). Hasta el momento no se cuenta con trabajos acerca del impacto de las invasiones de especies exóticas sobre el banco de semillas en los pastizales naturales serranos del Sistema de Tandilia. El objetivo del presente trabajo fue caracterizar y comparar (a través de la abundancia total, la específica y por grupos funcionales, la diversidad, el valor zootécnico y la disimilitud florística) el banco de semillas germinable del suelo de dos ambientes: los pastizales de la RNP dominados por P. quadrifarium (pastizal 
natural) y los pastizales invadidos por $R$. melanoxylon (pastizal invadido).

\section{Materiales y Métodos}

\section{Descripción del sitio de estudio}

El estudio se realizó en un sector de la Reserva Natural Paititi, ubicada en el partido de General Pueyrredón, provincia de Buenos

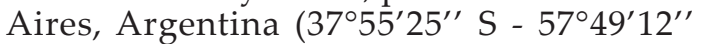
O), correspondiente a la ecorregión Pampa Austral (Miñarro and Bilenca 2008). La RNP consta de dos sierras: Sierra Grande y Sierra Chica (Figura 1). El clima de la zona es subhúmedo-húmedo, frío en invierno y caluroso en verano. La temperatura media es de $14{ }^{\circ} \mathrm{C}$, con máximas de $32{ }^{\circ} \mathrm{C}$ en verano y mínimas bajo cero en invierno. El promedio de las precipitaciones anuales acumuladas es de $850 \mathrm{~mm}$. Por lo general, en verano se registran períodos con déficit hídrico (Echeverría et al. 2017).

En el mes de mayo del año 2019 se determinó el área invadida por los árboles de $R$. melanoxylon en un sector de Sierra Chica mediante el análisis de imágenes satelitales y con el apoyo de información de campo obtenida recorriendo el sector a pie con un navegador personal GPS (Figura 1). Esto permitió diferenciar las zonas invadidas

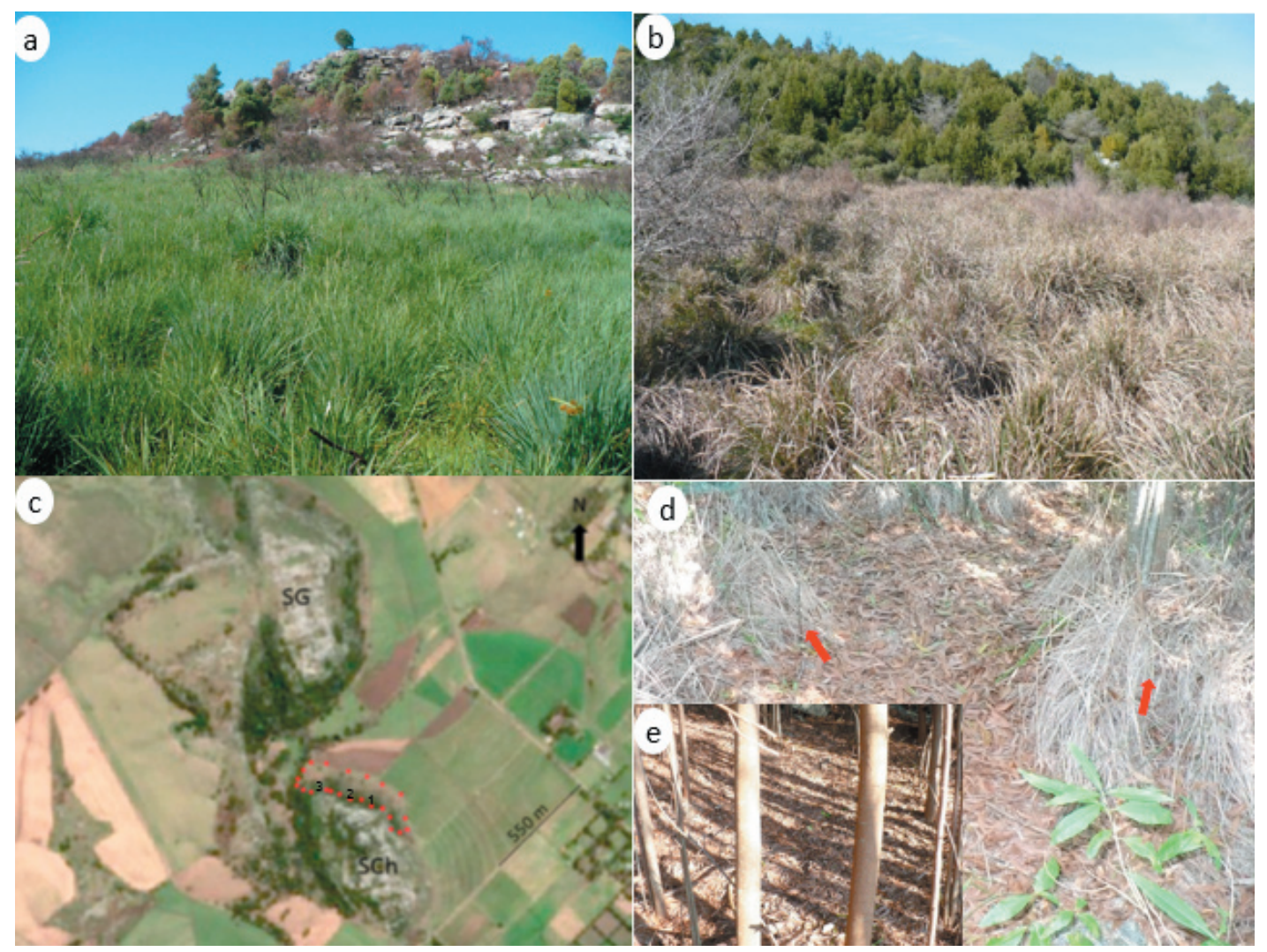

Figura 1. Reserva Natural Paititi (General Pueyrredón, Buenos Aires, Argentina). (a) Imagen tomada en mayo de 2014 del pastizal natural después de la quema en enero de 2014. (b) El pastizal natural de Paspalum quadrifarium Lam. (frente) y el pastizal invadido por R. melanoxylon (R. Br.) Pedley (fondo). (c) Vista satelital de Sierra Chica (SCh) y Sierra Grande (SG); los puntos rojos en Sierra Chica indican el área de estudio de invasión por Racosperma melanoxylon (R. Br.) Pedley y los números 1, 2 y 3 indican los sitios de muestreo del banco de semillas. (d) Plantas de P. quadrifarium muertas en el pastizal invadido (indicadas con flechas rojas). (e) Detalle del pastizal natural invadido por R. melanoxylon, con hojas secas acumuladas en el suelo y sin vegetación herbácea. La imagen satelital fue obtenida de Sentinel Playground (Sentinel Playground 2019).

Figure 1. Paititi Nature Reserve (General Pueyrredón, Buenos Aires, Argentina). (a) Photography of the natural grassland in May 2014 after burnt in January 2014. (b) Paspalum quadrifarium Lam. natural grassland (front) and invaded grassland by R. melanoxylon (R. Br.) Pedley (background). (c) Satellite image of Sierra Chica (SCh) and Sierra Grande (SG); the red dots in Sierra Chica correspond to the area studied of invasion by Racosperma melanoxylon (R. Br.) Pedley, and the numbers 1, 2 and 3 indicate the sampling sites for soil seed bank. (d) Dead P. quadrifarium plants in the invaded grassland (indicated by red arrows). (e) Natural grassland invaded by $R$. melanoxylon with dry leaves accumulated on the ground and without herbaceous vegetation. Satellite image obtained from Sentinel Playground (Sentinel Playground 2019). 
de otro tipo de vegetación como Baccharis dracunculifolia DC. Ssp. tandilensis (Spreg) Giuliano y los pajonales de P. quadrifarium. Con el programa QGIS (QGIS Development Team 2019) se trazó una grilla con celdas de $20 \times 20 \mathrm{~m}$ sobre la imagen satelital de la zona de estudio, se contaron las celdas ocupadas por los árboles y se determinó el porcentaje invadido respecto al número total de celdas; $35 \%$ estaba cubierto por acacias (Figura 1). A partir de esa información se establecieron tres sitios de muestreo con una fisonomía comparable entre los árboles de $R$. melanoxylon.

En cada sitio de muestreo se determinaron dos ambientes, el pastizal natural y el pastizal invadido por $R$. melanoxylon (Figura 1). Al momento de realizar el muestreo del banco de semillas, el ambiente del pastizal natural estaba dominado por $P$. quadrifarium. Si bien no se caracterizó la biomasa aérea de los sitios, trabajos previos en la reserva la estimaron en $\sim 16000 \mathrm{~kg} / \mathrm{ha}$, sin tener en cuenta el mantillo acumulado (Garavano 2018). Nuestros trabajos en el campo (Garavano 2018) y las imágenes satelitales nos permiten estimar que la invasión comenzó luego del incendio acontecido a principios del año 2014, por lo que, al momento de tomar las muestras de suelo, los árboles no tendrían más de cinco años de edad (Figura 1). El suelo del pastizal invadido estaba cubierto con hojas secas de los árboles. Se observaron algunas plantas aisladas de Dactylis glomerata L. con biomasa verde y las de $P$. quadrifarium muertas (Figura 1).

\section{Muestreo del banco de semillas del suelo y variables analizadas}

Para evaluar el banco de semillas germinable de ambos ambientes se utilizó el método de conteo por emergencia de plántulas. El 30 abril de 2019, luego de la época de dispersión primaria de semillas, se realizó un muestreo del banco en los dos ambientes de los tres sitios (Figura 1). Como el pastizal natural y el invadido tenían un límite en común en cada sitio, se dejó un borde de $\sim 3 \mathrm{~m}$ sin muestrear a cada lado de dicho límite para minimizar efectos de borde.

Para tomar muestras de suelo se utilizó un muestreador metálico de $2.5 \mathrm{~cm}$ de diámetro por $5 \mathrm{~cm}$ de profundidad (tarugos de 24.53 $\mathrm{cm}^{3}$ ). En los primeros $5 \mathrm{~cm}$ de suelo se encuentra el banco de semillas transitorio y, en pastizales, la mayor cantidad de semillas
(Márquez et al. 2002). No se incluyeron las porciones superficiales de hojas secas de acacias ni de otras plantas del pastizal (mantillo). En cada sitio se tomaron 10 muestras compuestas, formadas por 6 tarugos cada una (submuestras), siguiendo una transecta paralela al límite común. Cada muestra fue tomada al azar, distanciadas $\sim 1 \mathrm{~m}$ entre sí. Se tomaron 60 muestras de suelo en total (10 muestras $\times 3$ sitios $\times 2$ ambientes). La superficie de muestreo de suelo de cada sitio fue de $294.37 \mathrm{~cm}^{2}$, resultando un volumen apropiado $\left(1471.87 \mathrm{~cm}^{3}\right)$ para detectar la mayoría de las especies en pastizales (Roberts 1981, citado por Haretche and Rodríguez 2006).

El 2 de mayo de 2019, las muestras de suelo fueron acondicionadas en bandejas $(20 \times 16 \times 4 \mathrm{~cm}$ de largo, ancho y profundidad, respectivamente) con la base perforada. Cada bandeja tenía un sustrato de arena de río sobre la cual se distribuyó una muestra de suelo (6 tarugos desmenuzados). Cada bandeja fue colocada dentro de otra bandeja más grande sin perforaciones $(17 \times 24 \times 5 \mathrm{~cm}$ de ancho, largo y profundidad, respectivamente), a la cual se le agregaba agua de lluvia para mantener húmedo el sustrato y el suelo. Las bandejas fueron acondicionadas en una jaula antiáfidos del banco de germoplasma de la EEA INTA Balcarce, y rotadas al azar de forma periódica. Como control de una posible contaminación se utilizaron cuatro bandejas adicionales con arena de río, pero sin agregar los tarugos de suelo, que se manejaron como el resto. Los valores promedio $( \pm \mathrm{EE})$ de temperatura máxima, mínima, humedad relativa máxima y mínima del aire, y radiación fotosintéticamente activa entre principios de mayo y mediados de diciembre 2019 dentro de la jaula antiáfidos fueron $28.62 \pm 2.70{ }^{\circ} \mathrm{C} ; 2.52 \pm 1.54^{\circ} \mathrm{C}$; $82.93 \pm 4.34 \%, 24.98 \pm 3.42 \%$ y $419.85 \pm 79.21 \mu \mathrm{mol} /$ $\mathrm{m}^{-2}$.día ${ }^{-1}$, respectivamente. Desde mediados de diciembre de 2019 en adelante, debido a las elevadas temperaturas en la jaula antiáfidos, las bandejas que aún tenían plántulas fueron dejadas al aire libre, protegidas del sol y de la posible contaminación con semillas.

El tamaño del banco de semillas germinable fue determinado mediante la cantidad acumulada de plántulas emergidas en cada una de las 64 bandejas ( 2 ambientes $\times 3$ sitios $\times 10$ repeticiones, más 4 controles) y se expresó como semillas $/ \mathrm{m}^{2}$. Para ello, semanalmente se identificaron las especies que emergían, eliminándolas de inmediato. Aquellas que no se pudieron identificar en estado vegetativo, se trasplantaron y se cultivaron en macetas 
hasta la floración. Las muestras se dejaron secar a medida que cesaba la emergencia. La emergencia de plántulas finalizó luego de 10 meses (en marzo de 2020). No se registró la emergencia de ninguna plántula en las bandejas control. En abril de 2020 se seleccionaron al azar 8 bandejas del pastizal natural y 9 del pastizal invadido, las cuales fueron regadas para observar si se producía un segundo pulso de emergencia (Haretche and Rodríguez 2006; Baskin and Baskin 2014). Cuarenta y cinco días después, a mediados de mayo de 2020, al no observar emergencia se dio por concluido el estudio.

Las especies fueron identificadas utilizando las claves taxonómicas de Cabrera (1965, 1967, 1968, 1970) y la información de la Flora Argentina (2018). También se clasificaron por su origen en exóticas, nativas o endémicas, y por sus propiedades en forrajeras y no forrajeras (Flora Argentina 2018). Además, las especies se agruparon en tres grupos funcionales: gramíneas, dicotiledóneas y graminoides (Ciperáceas y Juncáceas). Para cada ambiente se calculó la densidad relativa de semillas (respecto al número total de semillas) de las especies nativas, exóticas, endémicas y de los grupos funcionales.

Se determinó el valor zootécnico (VZ) de cada ambiente, una medida de la calidad de su forraje para el ganado vacuno, utilizando la función:

$$
\mathrm{VZ}=\sum \mathrm{p}_{\mathrm{i}} \times \mathrm{q}_{\mathrm{i}}
$$

donde $\mathrm{p}_{\mathrm{i}}$ es la proporción de cada especie en un ambiente y $\mathrm{q}_{\mathrm{i}}$ es el valor de calidad forrajera de la especie (Rodríguez et al. 2016; Carro et al. 2019). Los valores de calidad oscilan entre 0 (mala forrajera) y 5 (excelente forrajera) (Rodríguez et al. 2016; Carro et al. 2019). Dado que no se cuenta con valor zootécnico en la bibliografía para Holcus lanatus L., Briza minor L. y Dactylis glomerata L., se les asignó un valor de 5 por ser consideradas excelentes forrajeras.

Se determinaron la riqueza (S), los índices de diversidad de Shannon $\left(\mathrm{H}=-\sum \mathrm{P}_{\mathrm{i}} \cdot \ln \left[\mathrm{P}_{\mathrm{i}}\right]\right)$, el de diversidad $\left(\mathrm{D}=1 / \sum\left(\mathrm{P}_{\mathrm{i}}\right)^{2}\right)$ y de equitatividad $(\mathrm{E}=\mathrm{D} / \mathrm{S})$ de Simpson, siendo $\mathrm{P}_{\mathrm{i}}$ el número de semillas de la especie i en un sitio dado dividido el número total de semillas en dicho sitio (Begon et al. 2006). Con la abundancia relativa de cada especie, calculada como su contribución respecto al número total de semillas en un ambiente, se elaboró un diagrama de rango-abundancia (Begon et al. 2006).

La riqueza específica se utilizó para estimar el índice de disimilitud florístico de Sorensen (IDS) entre ambientes (Baselga 2010), según la siguiente función:

$$
\operatorname{IDS}=(\mathrm{a}+\mathrm{b}) /(2 \mathrm{c}+\mathrm{a}+\mathrm{b})
$$

donde a=número de especies exclusivas registradas en el suelo del pastizal natural; $b=$ número de especies exclusivas registradas en el suelo del pastizal invadido y c=número de especies comunes para ambos ambientes.

\section{Análisis de los datos}

Las diferencias entre ambientes según las variables consideradas fueron analizadas mediante pruebas de diferencias de medias $\mathrm{t}$ de Student como muestras apareadas (tres sitios). En todos los casos se consideraron significativas las diferencias de medias asociadas con un $\mathrm{P}<0.05$. Los datos expresados en porcentajes fueron transformados antes de su análisis como raíz cuadrada arcoseno. Se verificó la normalidad de la distribución de los residuos mediante la prueba de ShapiroWilk. Las medias se presentan con su error estándar. Para todos los análisis estadísticos se utilizaron funciones del programa $R$ ( $R$ Development Core Team 2016).

\section{Resultados}

\section{Diversidad del banco de semillas}

Las primeras plántulas con los cotiledones desplegados fueron observadas en las muestras de ambos ambientes aproximadamente 16 días después de dar comienzo el estudio. En ambos ambientes se identificó un total de 61 especies, pertenecientes a 24 familias (Tabla 1). Cinco de esas familias estuvieron ausentes en el pastizal natural (Chenopodiaceae, Gentianaceae, Geraniaceae, Urticaceae y Verbenaceae) y siete en el pastizal invadido (Amaranthaceae, Brassicaceae, Campanulaceae, Iridaceae, Orobanchaceae, Primulaceae y Violaceae) (Tabla 1). En los dos ambientes, las familias mejor representadas fueron Asteraceae $(29.50 \%)$ y Poaceae $(13.11 \%)$. Las especies endémicas encontradas fueron cinco: Baccharis dracunculifolia, Gamochaeta argentina Cabrera, Senecio selloi (Spreng.) DC., Solanum chenopodiodes Lam. y Vicia nana Vogel (Tabla 1). Cynodon dactylon (L.) Pers. emergió a partir de pequeños fragmentos de rizomas y representó 
Tabla 1. Especies identificadas en el banco de semillas germinable de los dos ambientes estudiados dentro de la Reserva Natural Paititi (General Pueyrredón, provincia de Buenos Aires, Argentina): el pastizal natural (PN) de Paspalum quadrifarium Lam. y el pastizal natural invadido (PI) por Racosperma melanoxylon (R. Br.) Pedley. Las especies están ordenadas de mayor a menor abundancia en el pastizal natural invadido. V.Z.: valor zootécnico de las especies; ${ }^{1}$ : especies endémicas de ecosistemas serranos de Buenos Aires (Echeverría et al. 2017).

Table 1. Identified species in the germinable soil seed bank at the two studied environments within the Paititi Natural Reserve (Buenos Aires, Argentina) natural grasslands of Paspalum quadrifarium Lam. (PN) and natural grassland invaded by Racosperma melanoxylon (R. Br.) Pedley. (PI). Species were sorted by their decreasing abundance in the invaded grassland. V.Z.: zootechnical value of each plant species; ${ }^{1}$ : endemic species of the mountain ecosystems of Buenos Aires (Echeverría et al. 2017).

\begin{tabular}{|c|c|c|c|c|}
\hline \multirow[b]{2}{*}{ Especies } & \multirow[b]{2}{*}{ Familia } & \multicolumn{2}{|c|}{ Ambientes } & \multirow[b]{2}{*}{ V.Z. } \\
\hline & & PN (\%) & PI (\%) & \\
\hline Phalaris angusta Nees ex Trin. & Poaceae & 0.003 & 0.140 & 4 \\
\hline Spergula ramosa (Cambess.) D. Dietr. var. ramosa & Caryophyllaceae & 0.135 & 0.136 & 0 \\
\hline Senecio selloi (Spreng.) DC. ${ }^{1}$ & Asteraceae & 0.005 & 0.131 & 0 \\
\hline Baccharis dracunculifolia DC. ssp. tandilensis (Speg.) Giuliano ${ }^{1}$ & Asteraceae & 0.038 & 0.083 & 0 \\
\hline Conyza sumatrensis (Retz.) E. Walker & Asteraceae & 0.050 & 0.056 & 0 \\
\hline Solanum pilcomayense Morong & Solanaceae & 0.016 & 0.055 & 0 \\
\hline Gamochaeta argentina Cabrera ${ }^{1}$ & Asteraceae & 0.001 & 0.052 & 0 \\
\hline Pseudognaphalium cheiranthifolium (Lam.) Hilliard and B. L. Burtt. & Asteraceae & 0.042 & 0.052 & 0 \\
\hline Holcus lanatus L. & Poaceae & 0.122 & 0.049 & 5 \\
\hline Conyza bonariesis (L.) Cronquist & Asteraceae & 0.022 & 0.032 & 0 \\
\hline Juncus bufonius L. var. buffonius & Juncaceae & 0.098 & 0.029 & 1 \\
\hline Racosperma melanoxylon $\mathrm{R}$. Br. & Fabeceae & 0.000 & 0.018 & 0 \\
\hline Poa sp. & Poaceae & 0.067 & 0.018 & 4 \\
\hline Silene antirrhina L. var. antirrhina & Caryophyllaceae & 0.000 & 0.018 & 0 \\
\hline Carex bonariensis Desf. ex Poir. var. bonariensis & Cyperaceae & 0.057 & 0.013 & 2 \\
\hline Solanum chenopodiodes Lam. ${ }^{1}$ & Solanaceae & 0.008 & 0.013 & 0 \\
\hline Cirsium vulgare (Savi) Ten. & Asteraceae & 0.020 & 0.010 & 0 \\
\hline Artemisia verlotiorum Lamotte & Asteraceae & 0.048 & 0.008 & 0 \\
\hline Dichanthelium sabulorum (Lam.) Gould and C. A. Clark var. sabulorum & Poaceae & 0.011 & 0.006 & 0 \\
\hline Physalis viscosa L. & Poaceae & 0.006 & 0.006 & 1 \\
\hline Vicia nana Vogel $^{1}$ & Fabaceae & 0.001 & 0.006 & 1 \\
\hline Carduus acanthoides L. & Asteraceae & 0.022 & 0.005 & 0 \\
\hline Galium aparine $\mathrm{L}$. & Rubiaceae & 0.006 & 0.005 & 0 \\
\hline Cyperus sp. & Cyperaceae & 0.007 & 0.004 & 0 \\
\hline Dactylis glomerata $\mathrm{L}$. & Poaceae & 0.000 & 0.004 & 5 \\
\hline Juncus sp. & Juncaceae & 0.008 & 0.004 & 1 \\
\hline Salpichroa origanifolia (Lam.) Baill. & Solanaceae & 0.000 & 0.004 & 2 \\
\hline Gamochaeta coarctata (Willd.) Kerguélen & Asteraceae & 0.047 & 0.003 & 0 \\
\hline Lathyrus crassipes Gillies ex Hook. and Arn. & Fabaceae & 0.000 & 0.003 & 0 \\
\hline Veronica sp. & Plantaginaceae & 0.001 & 0.003 & 0 \\
\hline Briza minor $\mathrm{L}$. & Poaceae & 0.006 & 0.002 & 5 \\
\hline Chenopodium album $\mathrm{L}$. & Chenopodiaceae & 0.000 & 0.002 & 0 \\
\hline Gamochaeta stachydifolia (Willd.) Cabrera & Asteraceae & 0.001 & 0.002 & 0 \\
\hline Mentha pulegium L. & Lamiaceae & 0.012 & 0.002 & 0 \\
\hline Paspalum quadrifarium Lam. & Poaceae & 0.007 & 0.002 & 1 \\
\hline Sonchus oleraceus L. & Asteraceae & 0.002 & 0.002 & 0 \\
\hline Centaurium pulchellum (Sw.) Druce & Gentianaceae & 0.000 & 0.001 & 0 \\
\hline Cotula australis (Sieber ex Spreng.) Hook. & Asteraceae & 0.011 & 0.001 & 0 \\
\hline Cyclospermum leptophyllum (Pers.) Britton and P. Wilon & Apiaceae & 0.020 & 0.001 & 0 \\
\hline Dichondra microcalyx (Hallier f.) Fabris & Convolvulaceae & 0.002 & 0.001 & 0 \\
\hline Geranium molle L. & Geraniaceae & 0.000 & 0.001 & 0 \\
\hline Parietaria debilis G. Forst. & Urticaceae & 0.000 & 0.001 & 0 \\
\hline Senecio madagascariensis Poir. & Asteraceae & 0.001 & 0.001 & 0 \\
\hline Verbena montevidensis Spreng. & Verbenaceae & 0.000 & 0.001 & 0 \\
\hline Agalinis communis (Cham. and Schltdl.) D'Arcy & Orobanchaceae & 0.003 & 0.000 & 2 \\
\hline Alternanthera philoxeroides (Mart.) Griseb. f. philoxeroides & Amaranthaceae & 0.001 & 0.000 & 0 \\
\hline Ambrosia tenuifolia Spreng. & Asteraceae & 0.012 & 0.000 & 0 \\
\hline Ammi majus L. & Apiaceae & 0.001 & 0.000 & 0 \\
\hline Anagallis arvensis $\mathrm{L}$. & Primulaceae & 0.020 & 0.000 & 0 \\
\hline Baccharis crispa Spreng. & Asteraceae & 0.001 & 0.000 & 0 \\
\hline Convolvulus arvensis $\mathrm{L}$. & Convolvulaceae & 0.001 & 0.000 & 0 \\
\hline Cynodon dactylon (L.) Pers. & Poaceae & 0.002 & 0.000 & 2 \\
\hline Helminthotheca echioide (L) Holub. & Asteraceae & 0.002 & 0.000 & 0 \\
\hline Ipomoea sp. & Convolvulaceae & 0.002 & 0.000 & 0 \\
\hline Lepidium didymum $\mathrm{L}$. & Brassicaceae & 0.001 & 0.000 & 0 \\
\hline Lolium multiflorum Lam. & Poaceae & 0.002 & 0.000 & 5 \\
\hline Sisyrinchium chilense Hook. ssp. Chilense & Iridaceae & 0.021 & 0.000 & 0 \\
\hline Taraxacum officinale G. Weber ex F. H. Wigg. & Asteraceae & 0.002 & 0.000 & 2 \\
\hline Trifolium repens $\mathrm{L}$. & Fabaceae & 0.002 & 0.000 & 4 \\
\hline Triodanis perfoliata (L.) Nieuwl. var. biflora (Ruiz and Pav.) T. R. Bradley & Campanulaceae & 0.001 & 0.000 & 0 \\
\hline Viola arvensis Murray ssp. arvensis & Violaceae & 0.002 & 0.000 & 0 \\
\hline
\end{tabular}


aproximadamente el $0.25 \%$ del total de las plántulas del pastizal natural (Tabla 1).

No se registraron diferencias significativas entre los ambientes en la riqueza de especies ni en la densidad del banco de semillas (Tabla 2). Los índices de equitatividad y de diversidad específica no variaron significativamente entre ambientes (Tabla 2). Si bien los valores medios del valor zootécnico tampoco fueron estadísticamente diferentes entre ambientes, el valor observado en el pastizal natural fue $26 \%$ mayor que en el pastizal invadido (Tabla 2). La densidad de semillas de cada grupo funcional no difirió significativamente entre los ambientes (Tabla 2). En ambos ambientes, el banco de semillas de las dicotiledóneas $(66.10 \%)$ fue más abundante que el de las gramíneas $(22.88 \%)$ y el de las graminoides (10.99\%). Tampoco difirieron significativamente entre ambientes el banco de semillas de las especies nativas $(77.87 \%$; $\mathrm{t}=-0.782$, g.l. $=2 ; \mathrm{P}=0.516)$ ni el de las exóticas (22.13\%; $\mathrm{t}=2.297 ; \mathrm{g} .1 .=2 ; \mathrm{P}=0.148)$.

Tabla 2. Valores medios ( \pm EE) de densidad del banco de semillas germinable, riqueza, grupos funcionales (dicotiledóneas, gramíneas y graminoides), índices de diversidad (D, y H de Shannon) y de equitatividad (E de Simpson), y valor zootécnico del pastizal natural (PN) de Paspalum quadrifarium Lam. y en el pastizal natural invadido (PI) por Racosperma melanoxylon (R. Br.) Pedley. Para cada variable, letras iguales indican diferencias no significativas entre ambientes $(\mathrm{P}<0.05)$.

Table 2. Average values $( \pm S E)$ of total density of the germinable seed bank, richness, functional groups (dicots, grass and graminoids), indices of diversity (D and Shannon's H) and equitability (Simpsons' E), and zootechnical value of Paspalum quadrifarium Lam. natural grassland (PN) and natural grassland invaded by Racosperma melanoxylon (R. Br.) Pedley (PI). For each variable, same letters indicate no significative differences between environments $(\mathrm{P}<0.05)$.

\begin{tabular}{|c|c|c|c|c|c|}
\hline \multirow[b]{2}{*}{ Variable } & \multicolumn{2}{|c|}{ Ambientes } & \multirow[b]{2}{*}{$\mathrm{t}$} & \multirow[b]{2}{*}{ g.1. } & \multirow[b]{2}{*}{$\mathrm{P}$} \\
\hline & $\mathrm{PN}$ & PI & & & \\
\hline Banco de semillas (nro. $/ \mathrm{m}^{2}$ ) & $9092.71 \pm 1725.95$ a & $10440.18 \pm 1989.54 \mathrm{a}$ & 0.369 & 2 & 0.747 \\
\hline Riqueza (nro. especies) & $32.33 \pm 2.40 \mathrm{a}$ & $28.66 \pm 1.45$ a & -0.157 & 2 & 0.256 \\
\hline Dicotiledóneas (nro. plantas $/ \mathrm{m}^{2}$ ) & $5389.94 \pm 1246.19$ a & $7586.69 \pm 383.49 \mathrm{a}$ & 1.705 & 2 & 0.230 \\
\hline Gramíneas (nro. plantas/m²) & $2140.12 \pm 643.04 \mathrm{a}$ & $2321.30 \pm 1396.00 \mathrm{a}$ & 0.092 & 2 & 0.934 \\
\hline Graminoides (nro. plantas $/ \mathrm{m}^{2}$ ) & $1536.63 \pm 1163.12$ a & $532.19 \pm 271.99 \mathrm{a}$ & -0.841 & 2 & 0.488 \\
\hline $\mathrm{H}$ & $2.78 \pm 0.14 \mathrm{a}$ & $2.54 \pm 0.10 \mathrm{a}$ & -1.013 & 2 & 0.417 \\
\hline $\mathrm{D}$ & $11.61 \pm 2.59 \mathrm{a}$ & $8.76 \pm 1.76 \mathrm{a}$ & -0.771 & 2 & 0.520 \\
\hline $\mathrm{E}$ & $0.36 \pm 0.09 \mathrm{a}$ & $0.30 \pm 0.05 a$ & -0.604 & 2 & 0.606 \\
\hline Valor Zootécnico & $1.22 \pm 0.18 \mathrm{a}$ & $0.89 \pm 0.37 \mathrm{a}$ & 0.732 & 2 & 0.540 \\
\hline
\end{tabular}

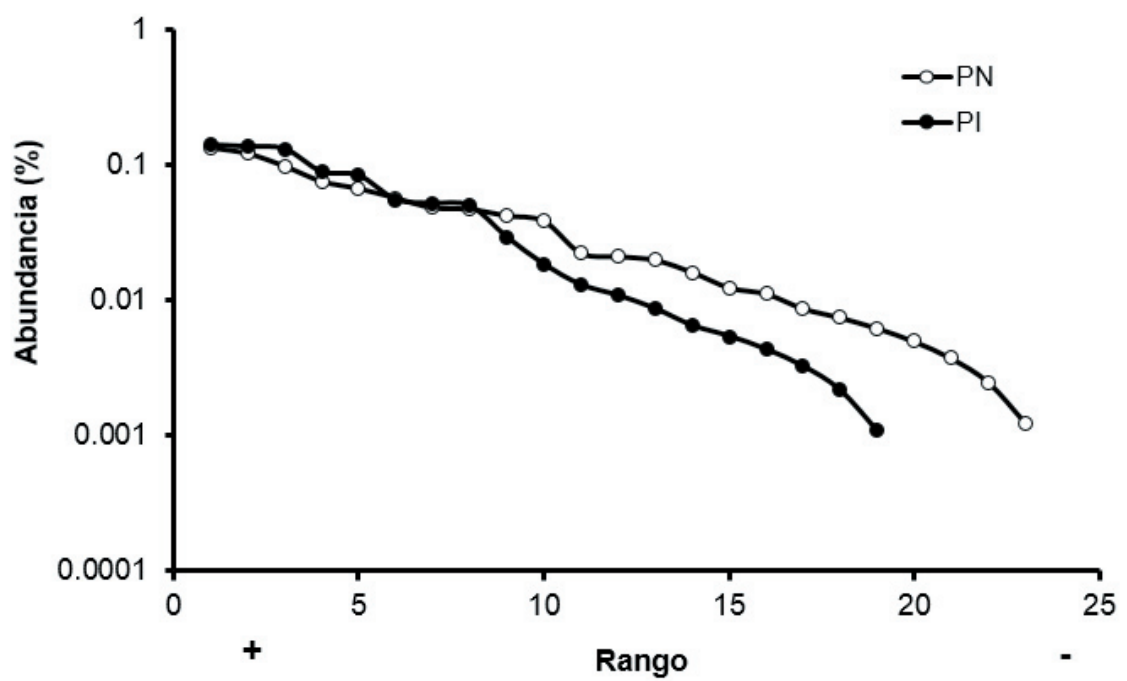

Figura 2. Diagrama de rango-abundancia de especies en los bancos de semillas germinables de los dos ambientes estudiados: el pastizal natural (PN) de Paspalum quadrifarium Lam. y el pastizal natural invadido (PI) de Racosperma melanoxylon (R. Br.) Pedley. Cada punto en la curva es el valor promedio de abundancia de los tres sitios de muestreo.

Figure 2. Rank-abundance patterns of the plant species in the germinable soil seed bank of the two studied environments: the Paspalum quadrifarium Lam. natural grassland (PN) and the natural grassland invaded by Racosperma melanoxylon (R. Br.) Pedley (PI). Each point on the curve is the average abundance of three sampling sites. 
Disimilitud entre ambientes y curvas de rangoabundancia

El índice de disimilitud florística entre ambientes fue $0.36 \pm 0.04$. Las curvas de rango-abundancia ponen en evidencia dos aspectos: que en el pastizal natural se registró el mayor número de especies y que las especies comunes variaron en su abundancia (Figura 2, Tabla 1). Del total de las especies identificadas, 10 estuvieron ausentes en el pastizal natural y 17 en el suelo del pastizal invadido (Tabla 1 ). Las semillas de las tres especies más abundantes fueron Phalaris angusta Nees ex Trin. (14.09\%), Spergula ramosa (Cambess.) D. Dietr. (13.66\%) y Senecio selloi $(13.12 \%)$ en el pastizal invadido; en cambio, en el pastizal natural fueron Spergula ramosa (13.50\%), Holcus lanatus L. (12.25\%) y Juncus bufonius L. var buffonius (9.87\%). El banco de semillas de $P$. quadrifarium estuvo mejor representado en el pastizal natural $(0.74 \%)$ que en el invadido $(0.21 \%)$ (Tabla 1$)$. El banco de semillas de $R$. melanoxylon representó el $1.84 \%$ del total de semillas del pastizal invadido, pero estuvo ausente en el pastizal natural (Tabla 1).

\section{DiscusióN}

La falta de diferencias significativas en la densidad, en los índices de diversidad y equitatividad, en el número de especies y en la abundancia de los grupos funcionales entre el banco de semillas germinable del pastizal natural y el del invadido podría deberse al escaso tiempo transcurrido desde la invasión. El banco de semillas de ambos pastizales fue más rico en especies de las familias Asteraceae y Poaceae, lo que es consistente con los relevamientos previos en la RNP (Echeverría et al. 2017; Garavano 2018). Si bien en este estudio no fueron incluidas las semillas retenidas en las hojas secas debajo de los árboles de acacias ni en el mantillo del pastizal, el banco de semillas germinable en los primeros $5 \mathrm{~cm}$ de profundidad del suelo es importante en el mantenimiento y regeneración de las poblaciones de la mayoría de las plantas de este tipo de comunidad, luego de disturbios como el fuego y el pastoreo (Márquez et al. 2002).

Si bien los bancos de semillas germinables de ambos ambientes fueron comparables, el índice de disimilitud florística y el rangoabundancia de las especies presentes ponen en evidencia diferencias entre los bancos analizados. Esas diferencias también se hicieron evidentes en la ausencia de algunas especies y familias, que fue mayor en el banco de semillas del pastizal invadido. Esto podría ser consecuencia del agotamiento del banco de semillas por los efectos de la vegetación dominante y la actividad de la fauna edáfica (Gioria and Osborne 2010; Le Maitre et al. 2011; Gioria et al. 2012). En la RNP se documentó que las hojas secas de las acacias forman un microambiente en el suelo que modifica la riqueza y la abundancia de la fauna edáfica (entre ellos, a las hormigas cortadoras) respecto a los ambientes no invadidos y dominados por especies como P. quadrifarium (Arcusa 2019). Las hormigas cortadoras pueden llevar a las semillas del suelo hasta el nido y utilizarlas como sustrato para cultivar el hongo (Montoya-Lerma et al. 2012). Los carábidos y los roedores que habitan en los ambientes de estudio incluyen en sus dietas a las semillas del banco del suelo, siendo un importante factor de regulación de la estructura de las comunidades vegetales (Murillo et al. 2007). Por otro lado, la liberación de sustancias alelopáticas y la competencia aérea y subterránea por parte de las plantas de $R$. melanoxylon pueden afectar negativamente el banco de semillas, el establecimiento y la supervivencia de algunas especies (Mack et al. 2000; Le Maitre et al. 2011; Gioria et al. 2012; Cuevas and Zalba 2013). Esto podría explicar nuestra observación de plantas muertas de $P$. quadrifarium en los pastizales invadidos (Figura 1). Otra diferencia registrada entre los ambientes fue que el banco del pastizal invadido se empobreció en cantidad de semillas de alta calidad forrajera, como las exóticas naturalizadas Lolium multiflorum y Trifolium repens o la gramínea Holcus lanatus, mientras que se incrementó la proporción de algunas especies tóxicas para el ganado, como Senecio selloi (Micheloud et al. 2017). Si bien tampoco fue una diferencia estadísticamente significativa, estos cambios resultaron en un valor zootécnico promedio $26 \%$ menor en el pastizal invadido que en el natural.

Si se recuperara la estructura del pastizal natural mediante el control de las acacias a través de corte, de tratamiento químico o de una combinación de ambos (Le Maitre et al. 2011; Cueva and Zalba 2013), P. quadrifarium no debería faltar en la comunidad vegetal restaurada. Los manejos del pastizal que afectan de manera negativa a su población conducen a la comunidad vegetal a un estado sucesional irreversible dominado por pastos cortos (Laterra et al. 1998). La importancia de los pajonales de $P$. quadrifarium radica en que 
no sólo son un recurso forrajero para el ganado, sino también refugio y fuente de alimento para la fauna local (Comparatore et al. 1996). La ausencia de plantas vivas de $P$. quadrifarium en los pastizales invadidos pone en evidencia que el restablecimiento de la población luego del control de las acacias quedaría condicionado por la densidad de su banco de semillas germinable en el suelo. Esta especie presenta un banco de semillas transitorio (Laterra et al. 1994) y encontramos que su banco germinable se redujo a aproximadamente un tercio luego del período de invasión por las plantas de $R$. melanoxylon (68.00 semillas $/ \mathrm{m}^{2}$ en el pastizal natural vs. 22.66 semillas $/ \mathrm{m}^{2}$ en el pastizal invadido). Dado que la densidad estimada de plantas adultas de $P$. quadrifarium en la RNP fue $1.47 \pm 0.08$ plantas $/ \mathrm{m}^{2}$ (Vignolio et al., datos no publicados), la cantidad de semillas registradas podría ser suficiente para restablecer a las plantas del pajonal luego del control de los árboles.

En conclusión, nuestros resultados sugieren que el tiempo desde la invasión por $R$. melanoxylon no ha sido suficiente para provocar diferencias mayores en los bancos de semillas. El pastizal invadido conservó un banco viable de semillas, entre ellas de algunas especies endémicas y amenazadas como Baccharis dracunculifolia y Senecio selloi (Echeverría et al.
2017). En tal sentido, es posible considerar que un control temprano de las plantas de acacias posibilitaría la recuperación del pastizal invadido a partir del banco de semillas del suelo, pudiendo obtener una estructura y composición florística comparables a la del pastizal natural. Cabe considerar que la RNP es manejada con pastoreo doméstico y que el banco de semillas de las especies tóxicas como S. selloi fue importante en el suelo del pastizal invadido. Por lo tanto, es recomendable, si se eliminan los árboles, hacer un relevamiento periódico de la flora que se va estableciendo para poder tomar decisiones de manejo sobre las especies indeseables.

Agradecimientos. A la Licenciada María Rosa Desirello por la lectura crítica del presente artículo, al dueño de la Reserva Natural Paititi, Esteban González Zugasti por facilitarnos el ecosistema para desarrollar el presente trabajo, y al Editor Asociado y los revisores de la revista Ecología Austral por sus comentarios. A la Dra. S. San Martino por la ayuda en estadística. El trabajo corresponde al proyecto financiado por la Facultad de Ciencias Agrarias (UNMdP. 15/A602; AGR 598/19). Núcleos de Actividades Científicas y Tecnológicas: Ecología de Poblaciones y Comunidades Vegetales en Ecosistemas Naturales e Intervenidos.

\section{Referencias}

Amiotti, N. M., P. Zalba, L. F. Sánchez, and N. Peinemann. 2000. The impact of single trees on properties of loessderived grassland soils in Argentina. Ecology 81:3283-3290. https://doi.org/10.1890/0012-9658(2000)081[3283. TIOSTO12.0.CO;2

Arcusa, J. M. 2019. Fire effects on the ant community in areas of native and exotic vegetation. Sociobiology 66:44-51. https://doi.org/10.13102/sociobiology.v66i1.3178.

Baldi, G., J. P. Guerschman, and J. M. Paruelo. 2006. Characterizing fragmentation in temperate South America grasslands. Agriculture, Ecosystems and Environment 116:197-208. https://doi.org/10.1016/j.agee.2006.02.009.

Baselga, A. 2010. Partitioning the turnover and nestedness components of beta diversity. Global Ecology and Biogeography 19:134-143. https://doi.org/10.1111/j.1466-8238.2009.00490.x.

Baskin, C. C., and Baskin, J. M. 2014. Seeds: Ecology, biogeography, and evolution of dormancy and germination. Segunda edición. Academic Press, New York, USA.

Begon, M., C. R. Townsend, and J. L. Harper. 2006. Ecology from individuals to ecosystems. Cuarta edición. Blackwell Publishing, Oxford, Reino Unido.

Cabrera, A. 1965. Flora de la Provincia de Buenos Aires. Tomo 4, Parte IV: Oxalidáceas a Umbelíferas. Colección Científica del INTA. Buenos Aires. Argentina.

Cabrera, A. 1967. Flora de la Provincia de Buenos Aires. Tomo 4, Parte III: Piperáceas a Leguminosas. Colección Científica del INTA. Buenos Aires. Argentina.

Cabrera, A. 1968. Flora de la Provincia de Buenos Aires. Tomo 4, Parte I: Pteridófitas - Gimnospermas y Monocotiledóneas. Colección Científica del INTA. Buenos Aires. Argentina.

Cabrera, A. 1970. Flora de la Provincia de Buenos Aires. Tomo 4, Parte II: Gramíneas. Primera edición. Colección Científica del INTA, Buenos Aires, Argentina.

Carranza, S. L. 2017. Revisión bibliográfica sobre Acacia melanoxylon: su silvicultura y su madera. Revista de la Facultad de Agronomía, La Plata 106:145-154.

Carro, M. E., A. Casal, D. Coria, G. J. Fernández, and M. S. Beade. 2019. Short-term plant community response to grassland management intended to improve forage quality for the endangered Pampas deer. Journal for Nature Conservation 50:125716.

Comparatore, V. M., M. M. Martínez, A. I. Vassallo, M. Barg, and J. P. Isacch. 1996. Abundancia y relaciones con el hábitat de aves y mamíferos en pastizales de Paspalum quadrifarium (paja colorada) manejados con fuego (Provincia de Buenos Aires, Argentina). Interciencia 21:228-237. 
Cuevas, Y. A., and S. M. Zalba. 2010. Recovery of native grasslands after removing invasive pines. Restoration Ecology 18:711-719. https://doi.org/10.1111/j.1526-100X.2008.00506.x.

Cuevas, Y. A., and S. M. Zalba. 2013. Efecto del tipo de corte y de tratamientos en el mantillo para la restauración de pastizales naturales invadidos por Pinus halepensis. Boletín de la Sociedad Argentina de Botánica 48:315-329. https: //doi.org/10.31055/1851.2372.v48.n2.6265.

Echeverría, M. L., S. I. Alonso, and V. M. Comparatore. 2017. Survey of the vascular plants of Sierra Chica, the untouched area of the Paititi Natural Reserve (southeastern Tandilia mountain range, Buenos Aires province, Argentina). Check List 13:1003-1036. https://doi.org/10.15560/13.6.1003.

Ferrari, A. E., and L. G. Wall. 2004. Utilización de árboles fijadores de nitrógeno para la revegetación de suelos degradados. Revista de la Facultad de Agronomía, La Plata 105:63-87.

Flora Argentina. 2018. Plantas vasculares de la República Argentina. URL: floraargentina.edu.ar.

Garavano, E. 2018. Estudio de Solanum commersonii Dunal en un ecosistema serrano del Sistema de Tandilia (Buenos Aires) para implementar su conservación in situ. Tesis M. Sc. Facultad de Ciencias Agrarias, Universidad Nacional de Mar del Plata, Argentina. Pp. 124.

Ghersa, C. M., E. de la Fuente, S. Suárez, and R. J. C. León. 2002. Woody species invasion in the Rolling Pampagrasslands, Argentina. Agriculture, Ecosystems and Environment 88:271-278. https://doi.org10.1016/S0167-8809(01)00209-2

Gioria, M., and B. Osborne. 2010. Similarities in the impact of three large invasive plant species on soil seed bank communities. Biological Invasions 12:1671-1683. https://doi.org/10.1007/s10530-009-9580-7.

Gioria, M., P. Pyšek, and L. Moravcová. 2012. Soil seed banks in plant invasions: Promoting species invasiveness and long-term impact on plant community dynamics. Preslia 84:327-350.

Haretche, F., and C. Rodríguez. 2006. Banco de semillas de un pastizal uruguayo bajo diferentes condiciones de pastoreo. Ecología Austral 16:105-113.

Hussain, M. I., L. González, and M. J. Reigosa. 2011. Allelopathic potential of Acacia melanoxylon on the germination and root growth of native species. Weed Biology and Management 11:18-28. https://doi.org/10.1111/j.14456664.2011.00401.x.

Laterra, P., L. Ricci, O. R. Vignolio, and O. Fernández. 1994. Efectos del fuego y del pastoreo sobre la regeneración por semillas de Paspalum quadrifarium en la Pampa Deprimida, Argentina. Ecología Austral 4:101-109.

Laterra, P., O. R. Vignolio, L. G. Hidalgo, O. N. Fernández, M. A. Cauhépé, and N. O. Maceira. 1998. Dinámica de pajonales de paja colorada (Paspalum spp.) manejados con fuego y pastoreo en la Pampa Deprimida Argentina. Ecotropicos 11:141-149.

Le Maitre, D. C., M. Gaertner, E. Marchante, E. J. Ens, P. M. Holmes, A. Pauchard, P. J. O’Farrell, A. M. Rogers, R. Blanchard, J. Blignaut, and D. M. Richardson. 2011. Impacts of invasive Australian acacias: implications for management and restoration. Diversity and Distributions 17:1015-1029. https://doi.org/10.1111/j.1472-4642.2011.00816.x.

Mack, R. N., D. Simberloff, W. M. Lonsdale, H. Evans, M. Clout, and F. A. Bazzaz. 2000_Biotic invasions:-causes epidemiology, global consequences, and control. Ecological Applications 10:689-710. https://doi.org/10.1890/10510761(2000)010[0689:BICEGC[2.0.CO;2

Márquez, S., G. Funes, M. Cabido, and E. Pucheta. 2002. Grazing effects on the germinable seed bank and standing vegetation in mountain grasslands from central Argentina. Revista Chilena de Historia Natural 75:327-337. https: //doi.org/10.4067/S0716-078X2002000200006.

Micheloud, J. F., P. Merep, R. H. Tomas, M. Perotti, and C. Schuff. 2017. Intoxicación de equinos por Senecio sp. en el noroeste argentino. Revista Veterinaria 28:126-131. https://doi.org/10.30972/vet.2822538.

Miñarro, F., and D. Bilenca. 2008. The conservation status of temperate grasslands in central Argentina. Special Report. Fundación Vida Silvestre Argentina. Buenos Aires.

Montoya-Lerma, J., C. Giraldo-Echeverri, I. Armbrecht, A. Farji-Brener, and Z. Calle. 2012. Leaf-cutting ants revisited: Towards rational management and control. International Journal of Pest Management 58:225-247. https://doi.org/ 10.1080/09670874.2012.663946

Murillo, N., P. Laterra, and G. Monterubbianesi. 2007. Post-dispersal granivory in_a_tall-tussock grassland.A positive feedback mechanism of dominant? Journal Vegetation Science 18:799-806. https://doi.org/10.1111/j.1654$1103.2007 . t \mathrm{~b} 02596 . x$

Pimentel, D. 2002. Biological invasions: economic and environmental costs of alien plant, animal, and microbe species. Primera edición. CRC Press, New York, USA. https://doi.org/10.1201/9781420041668.

QGIS Development Team. 2019. QGIS Geographic Information System. Open Source Geospatial Foundation Project. URL: ggis.org

R Development Core Team. 2016. R: A language and environment for statistical computing. R Foundation for Statistical Computing, Vienna, Austria. URL: R-project.org.

Renuka, G., M. S. Rao, V. Praveen Kumar, M. Ramesh, and S. R. Reddy.2012.Arbuscular mycorrhizal dependency of Acacia melanoxylon R. Br. Proceedings of the National Academy of Sciences, India Section B: Biological Sciences 82: 441-446. https://doi.org/10.1007/s40011-012-0025-1.

Rodríguez, A., E. Jacobo, G. Roitman, F. Miñarro, P. Preliasco, and M. Beade. 2016. Manejo de la oferta forrajera en el Parque Nacional Campos del Tuyú y en campos ganaderos vecinos para la conservación del venado de las pampas. Ecología Austral 26:150-165. https://doi.org/10.25260/EA.16.26.2.0.152.

Sala, O. E., L. Yahdjian, K. Havstad, and M. R. Aguiar. 2017. Rangeland ecosystem services: Nature's supply and humans' demand. Pp. 467-489 en D. D. Briske (ed.). Rangeland Systems. Springer. https://doi.org/10.1007/978-3-31946709-2_14.

Souza-Alonso, P., J. Rodríguez, L. González, and P. Lorenzo. 2017. Here to stay. Recent advances and perspectives about Acacia invasion in Mediterranean areas. Annals of Forest Science 74: 55. https://doi.org/10.1007/s13595-017-0651-0.

Zalba, S. M., and C. B. Villamil. 2002. Woody plant invasion in relictual grasslands. Biological Invasions 4:55-72. https: //doi.org/10.1023/A:1020532609792. 